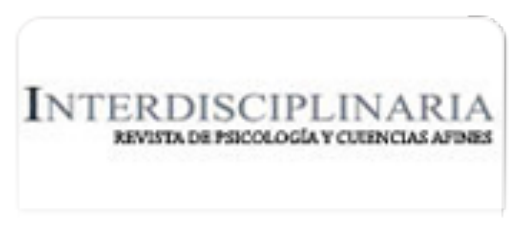

Interdisciplinaria

ISSN: 0325-8203

ISSN: 1668-7027

interdisciplinaria@fibercorp.com.ar

Centro Interamericano de Investigaciones Psicológicas y

Ciencias Afines

Argentina

Godoy, María Pía; Sepúlveda, José; Araneda, Lorena;

Canario, Rubén; Fonseca, Javier; Sáez, Carlos

Actitud hacia el consumo, ahorro y endeudamiento en

titulados de una universidad pública del sur de Chile

Interdisciplinaria, vol. 35, núm. 2, 2018, Julio-, pp. 511-525

Centro Interamericano de Investigaciones Psicológicas y Ciencias Afines

Argentina

Disponible en: https://www.redalyc.org/articulo.oa?id=18058785016

Cómo citar el artículo

Número completo

Más información del artículo

Página de la revista en redalyc.org

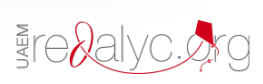

Sistema de Información Científica Redalyc Red de Revistas Científicas de América Latina y el Caribe, España y Portugal Proyecto académico sin fines de lucro, desarrollado bajo la iniciativa de acceso abierto 


\title{
Actitud hacia el consumo, ahorro y endeudamiento en titulados de una universidad pública del sur de Chile*
}

\section{Attitudes toward purchase, saving and indebtedness in university graduates from a public university of southern Chile}

\author{
María Pía Godoy**, José Sepúlveda***, Lorena Araneda****, \\ Rubén Canario*****, Javier Fonseca****** y Carlos Sáez*******
}

\footnotetext{
*Esta investigación está adscrita al proyecto de investigación DIUFRO DI13-0034

**Magister en Psicología. Académico del Departamento de Psicología e investigador del Núcleo Científico y

Tecnológico en Ciencias Sociales y Humanidades de la Universidad de La Frontera. E-mail: mariapia.godoy@ufrontera.cl

***Magister en Psicología. Académico del Departamento de Psicología e investigador del Núcleo Científico y Tecnológico en Ciencias Sociales y Humanidades de la Universidad de La Frontera.

****Licenciada en Psicología. Estudiante de la Carrera de Psicología de la Universidad de La Frontera.

*****Licenciado en Psicología. Estudiante de la Carrera de Psicología de la Universidad de La Frontera.

******Licenciado en Psicología. Estudiante de la Carrera de Psicología de la Universidad de La Frontera.

*******Licenciado en Psicología. Estudiante de la Carrera de Psicología de la Universidad de La Frontera.
}

Universidad de la Frontera (UFRO). Temuco, Chile.

\section{Resumen}

La presente investigación tiene por objeto comparar las actitudes hacia el ahorro, consumo y endeudamiento en titulados de una universidad pública del sur de Chile, a partir de su género, nivel socioeconómico y facultad de procedencia. Cuenta con un diseño cuantitativo, no experimental, ex post facto, de alcance descriptivo-correlacional y de tipo transversal. Se administraron la Escala de Actitudes hacia el Endeudamiento, la Escala de Actitudes hacia el Ahorro, la Escala de Actitudes hacia la Compra y la Escala de Evaluación del Nivel Socioeconómico ESOMAR. Además, se aplicó un cuestionario de preguntas de clasificación sociodemográfica, que fue respondido por 120 titulados universitarios de una universidad pública del sur de Chile contactados mediante el método bola de nieve. Se realizaron análisis descriptivos, pruebas de comparación de medias y medianas para dos muestras independientes para las compara- ciones según género, nivel socioeconómico y facultad de procedencia (pruebas $t$ para muestras independientes y pruebas $U$ de Mann-Whitney), y pruebas de correlación $r$ de Pearson para identificar posibles asociaciones entre las distintas escalas y subescalas. Los resultados permiten concluir un perfil esperable en cuanto a actitudes hacia el ahorro, endeudamiento y la compra, en titulados universitarios. En relación a las comparaciones, si bien se encontraron algunas diferencias significativas en cuanto a género y nivel socioeconómico de los participantes, resultaron más bien bajas. Finalmente, se hallaron asociaciones entre actitudes hacia el ahorro con austeridad y con racionalidad en la compra. También se encontraron asociaciones entre compulsividad en la compra con austeridad (inversamente) y con impulsividad en la compra, lo que apunta a una coherencia teórica de los constructos y sus dimensiones. Limitaciones del estudio incluyen el uso de medidas de autorreporte, lo que restringe la extrapolación de resultados a contextos 
fuera del presente estudio. Otra limitación corresponde al método de muestreo utilizado (bola de nieve) y tamaño muestral, lo que no permitiría generalizar los resultados a la población. A partir de los hallazgos y limitaciones, se realizan sugerencias para futuras investigaciones en alfabetización económica.

Palabras clave: Actitudes; Ahorro; Consumo; Compra; Endeudamiento; Titulados universitarios.

\section{Abstract}

Since the decade of 1970 onward in Chile, there has been a substantial change in terms of economic behaviour, which stems mainly from the installation of a neoliberal socioeconomic model. The profound consequences of this change for the Chilean society can be seen to this day, 40 years later, and these consequences include: broader access to goods and services for segments of the population that were previously excluded and/or that were a privilege of the economic elites; the assignation of new meanings to the concepts of purchase; and saving and indebtedness. These are all key concepts for the development and comprehension the neoliberal model. Complementary, the study of university graduates' behaviour is especially relevant in this area, considering that higher education is often thought to play a substantial role in social mobility. In the Chilean context, the transformations of the educational system have favoured the expansion of the admission numbers up to the point that seven of ten Chileans that pursue a graduate degree are the first members of their family to reach this educational level.

Considering the above, the present study aims to compare attitudes towards purchase, saving and indebtedness in graduates from a university in southern Chile, by gender, socioeconomic level and faculty.

The study follows a quantitative, cross-sectional, non-experimental, ex-post-facto design, with a descriptive-correlational scope. A questionnaire was completed by 120 participants contacted via snowball method. The questionnaire included: the Attitudes toward Indebted- ness Scale; Attitudes toward Saving Scale; and Attitudes toward Purchase Scale, a scale that evaluates the participants' socioeconomic level and other sociodemographic information to help describe the sample. Descriptive analyses were carried out, along with mean and median comparisons for gender, socioeconomic level and faculty ( $t$ tests for independent samples and Mann-Whitney $U$ tests). Finally, correlation tests with Pearson's $r$ test were carried out to test associations between the scales and subscales.

Results showed a profile that is desirable in university graduates in terms of attitudes towards saving, indebtedness and purchase, that is: favourable attitudes toward saving, austerity (a subscale of attitudes toward indebtedness) and rationality (a subscale of attitudes toward purchase), as well as negative attitudes toward hedonism (a subscale of attitudes toward indebtedness), and compulsivity and impulsivity (subscales of attitudes toward purchase). In terms of comparison, differences were found when comparing participants by gender in terms of attitudes toward compulsivity, and by their socioeconomic level by attitudes toward austerity in the indebtedness. Both these differences, although significant, were low when considering their effect size. No differences were found when comparing the faculty to which participants belonged in terms of attitudes toward saving, indebtedness and purchase. Finally, results showed associations between attitudes toward saving and austerity and with rationality, and between compulsivity and austerity (inversely) and with impulsivity. These results highlight the need to strengthen the attitudinal aspect in higher education environments where economic literacy is carried out, aiming to prepare students for their insertion into the workforce and their role as active citizens.

Limitations of this study include the subjective self-report measurements used, which are susceptible to the effect of social desirability. This limitation hinders the extrapolation of the results in terms of attitudes into actual behaviour in the real world. Another limitation comes from the sampling method used (snowball) and sample size, which does not allow to generalize the results to the population. Dra- 
wing from both findings and limitations, suggestions for future research in the subject of economic literacy and related issues are outlined.

Keywords: Attitudes; Saving; Consumption; Purchase; Indebtedness; University graduates.

\section{Introducción}

En Chile existe un antes y un después en el comportamiento económico y social a partir de la década del 70 (Carrillo, 2010). De acuerdo con Tironi (1999), este cambio se debería, en gran medida, a la instalación del modelo económico neoliberal que produjo profundos cambios en la sociedad chilena, como la ampliación en el acceso a bienes y servicios a gran parte de la población que hasta ese momento estaban vedados o eran privilegio de las elites económicas. En palabras de Ortiz (2002), este nuevo modelo trajo consigo una oportunidad de consumo nunca antes observada, tanto por los civiles como por las autoridades de la época.

Un análisis más profundo de la instalación del modelo económico neoliberal en Chile permite esclarecer los cambios sociales asociados a dicho fenómeno cuyas consecuencias, incluso 40 años después de iniciado dicho proceso, se viven en el día a día. De acuerdo con Denegri y Martínez (2004), una de las consecuencias de la instalación de este nuevo modelo económico es la ampliación del significado de las prácticas de consumo, ahorro y endeudamiento, desde una forma de satisfacer necesidades básicas a su instauración como articuladores de significaciones culturales y sociales, reguladores de emociones y formas de construir estatus en un entorno crecientemente cambiante.

De manera similar, Lipovetsky (2002) afirma que de la mano del modelo económico neoliberal, se ha instaurado una nueva era del consumo que involucra transformaciones en la sociedad actual, en cuanto a la vida personal y familiar como en los pro- cesos de socialización y educación de los nuevos miembros de la sociedad. Esta era del consumo se caracteriza por la generación y difusión de una cultura sustentada en las necesidades e informaciones, modificando lo estable y cotidiano, permitiendo la expansión de valores hedonistas y relegando conductas económicas predominantemente austeras. Como cualquier cambio cultural, esto implicaría cambios en el modo de relacionarse con el entorno, con los otros y con uno mismo, aceptando el nuevo paradigma como parte inherente de la vida de las personas.

En este contexto, resulta relevante explorar las actitudes que personas inmersas en este modelo económico sostienen en cuanto a ahorro, compra, consumo y endeudamiento, conceptos clave para la correcta administración de recursos personales y la disminución del riesgo en operaciones financieras.

\section{Consumo, ahorro y endeudamiento}

Existen tres conceptos clave para la instauración y comprensión del modelo neoliberal: ahorro, compra o consumo y endeudamiento. Dichos conceptos son clave pues se refieren a la administración de los recursos que permiten a los sujetos acceder a bienes y servicios. El consumo y/o compra estaría referido a la adquisición y uso de bienes y/o servicios a cambio de recursos; complementariamente, el ahorro permite la acumulación de recursos, ya sea con fines específicos o para eventualidades, haciendo referencia a una preparación para el futuro (cierto o incierto), mientras que el endeudamiento hace referencia al uso de recursos en nombre de otra persona o institución, usualmente con el fin de adquirir bienes o servicios. El estudio de estos conceptos resulta relevante por cuanto permite describir el comportamiento de los sujetos en este ecosistema neoliberal.

Catalán (2010) señala que el consumo coordina y posee un rol trascendental en la reproducción sistémica, en la integración, estratificación social, formación y cons- 
trucción de las identidades, así como en la selección y consecución de prácticas socialmente relevantes. La prevalencia de la socialización de los jóvenes por medio del ambiente externo a cambio del tradicional familiar, ocurre por medio de nuevas formas de interacción social donde la publicidad, la moda, la constante generalización de la educación, el impacto de los medios de comunicación de masas media y el aumento del capital adquisitivo, alcanzan especial importancia en sus concepciones y proyectos de vida.

De esta forma, el consumo en la sociedad chilena adquiere una gran relevancia en las formas de vida como en el desarrollo social de la juventud y se expresa en el incremento de las deudas de los jóvenes en los últimos años (INJUV, 2003, 2007, 2009). Una posible explicación para esta tendencia es el vínculo percibido entre éxito personal y profesional con el acceso a bienes y servicios por medio del consumo (Duarte, 2009). En esta propuesta, el consumo de bienes y servicios serían percibidos como indicadores de logro y realización de cada persona, lo que explicaría en parte la instauración de la era del consumo propuesta por Lipovetsky (2002).

En cuanto al ahorro, existe cierta indefinición teórica sobre cuál es la conducta que se podría esperar a lo largo de la vida. Una posible caracterización de dicho modelo es la Teoría del Ciclo Vital propuesta por Modigliani (1966, 1986), la cual presupone un nivel preferido o deseado de gasto, que es estable en el tiempo, y para una renta real del trabajo igualmente estable a lo largo del período de actividad laboral. Bajo estos supuestos, los hogares ahorrarían al inicio y mitad de la vida laboral, con objeto de acumular riqueza que sería utilizada en la etapa de retiro cuando la renta laboral es nula. Bajo este modelo, sería esperable que las personas jóvenes realicen un esfuerzo de acumulación de activos, mientras que los retirados actuarían en sentido contrario.

En cuanto al endeudamiento, si bien la flexibilización del concepto y actitudes hacia el endeudamiento ha permitido un ma- yor consumo y desarrollo económico, al mismo tiempo ha aumentado el riesgo de las operaciones financieras especialmente en las poblaciones más vulnerables como son los sectores de menores ingresos y particularmente la población joven, donde se observan comportamientos más consumistas (Denegri, Iturra, Palavecinos \& Ripoll, 1999; McElprang, Haskell \& Jenkins, 2009; Vidal, 1997). En la juventud, y producto de la necesidad de consolidación de la identidad, el consumo se convierte en una vía de acceso a un conjunto de símbolos y signos que favorecen la autorrealización y posibilitan la incorporación a una comunidad de iguales y por lo tanto, participa fuertemente de los procesos de construcción de identidad (PNUD/INJUV, 2003). De esta forma, el endeudamiento se ha convertido en un fenómeno de amplia aceptación social, donde los nuevos formatos que ha adquirido el dinero, unidos a nuevos patrones de consumo, han llevado incluso a proponer la existencia de una cultura del endeudamiento (Lea, Webley \& Levine, 1993).

Una forma de abordar el estudio del consumo, ahorro y endeudamiento es a través de las actitudes. De acuerdo a lo planteado por Luna-Arocas (1998), las actitudes serían un factor relevante en el complejo proceso del acceso al consumo por medio del endeudamiento y estarían relacionadas con las características personales del consumidor, incluyendo el manejo del dinero y su relación con el tipo de decisiones del consumidor frente a la compra. Las actitudes personales hacia la deuda establecerían lo que se denomina propensión a la deuda, lo cual antecede a la conducta real de deuda. En este plano se describen dos estilos actitudinales hacia el endeudamiento, uno caracterizado por una actitud más bien austera, marcada por el ahorro y, por otro lado, un estilo actitudinal marcadamente hedonista, en el que predominan actitudes positivas hacia el uso del crédito y el endeudamiento (Denegri, 1998).

En resumen, resulta interesante explorar las actitudes hacia el consumo, ahorro y endeudamiento, considerando el impacto que 
podrían tener en la comprensión del mundo económico que rodea y del que son parte las personas.

\section{Profesionales universitarios y consumo}

El estudio de la población universitaria resulta especialmente relevante teniendo en cuenta que la educación superior es considerada un factor sustancial en la generación de movilidad social (Espinoza \& González, 2010). En el caso de Chile, las transformaciones del sistema de educación superior han favorecido su expansión a tal punto que siete de cada diez jóvenes que estudian una carrera universitaria provienen de familias en las cuales ninguno de sus miembros tuvo esa oportunidad (Armanet, 2005) y el $80 \%$ de los estudiantes de educación superior ha logrado superar el nivel educacional conseguido por sus padres (Meneses, Rolando, Valenzuela \& Vega, 2010).

En la investigación del ahorro, consumo $\mathrm{y}$ endeudamiento en población universitaria, estudios previos en estudiantes universitarios chilenos evidencian una compleja relación entre la comprensión de la economía a nivel conceptual y la instalación de competencias para un comportamiento económico eficiente (Ayllón, Vallejos \& Yánez, 1999; Baeza, Durán \& Vargas, 1999; Medina, Méndez, Pérez \& Quezada, 1999). Ello indica que la educación económica conceptual resulta insuficiente por sí misma para modelar hábitos y actitudes hacia el consumo (Denegri, Gempp, Del Valle, Etchebarne \& González, 2006).

En este mismo sentido, datos de la VI Encuesta Nacional de la Juventud (INJUV, 2009) revelan que los jóvenes chilenos presentan un importante nivel de endeudamiento, presentando los mayores índices jóvenes pertenecientes a niveles socioeconómicos medios. Un ejemplo claro es que en los jóvenes que declaran ingresos propios, en los niveles de endeudamiento alcanzan niveles preocupantes, debido a los elevados montos promedio de lo adeudado. Asimismo, su participación en el mercado se ha construido en base al endeudamiento, de modo que jóvenes con ingresos propios de sectores más pobres muestran magnitudes de deuda en algunos puntos comparables con los de grupos más ricos, siendo que éstos disponen de mayores recursos para cubrir esas deudas. Es importante señalar, dentro de esta misma línea argumentativa, que jóvenes titulados universitarios se encuentran endeudados no solo por sus necesidades diarias, sino también por el gasto en su educación. Estadísticamente, el número de deudores y el monto aumentan considerablemente cada año; mientras que en el 2009 había 292.000 deudores con una suma de 901 millones de pesos, al 2013 el número de deudores ascendió a 481.000 y la deuda alcanzó los 1.820 millones de pesos (Toro, 2014).

Otro aspecto importante a considerar son los antecedentes expuestos por López y Schneider (2007) sobre los niveles de endeudamiento en estudiantes de la Universidad de la Frontera, evidenciando que un $52.2 \%$ de los participantes utilizaban medios de pago distintos al efectivo, como tarjetas de crédito de casas comerciales (79.5\%) y que 4 de cada 10 jóvenes de la muestra estaba endeudado, de los cuales las mujeres se encontraban levemente más endeudadas $(50.3 \%)$. Los resultados generales indicaron un $47.5 \%$ de endeudamiento, lo que constituye una cifra superior a la informada por el estudio del INJUV para el mismo año.

Siempre en el estudio de jóvenes estudiantes y titulados universitarios, la variable género debe ser tomada en consideración, pues antecedentes teóricos la vinculan a valores asociados al consumo (Borrás, 2007). Esta asociación apunta al imaginario social que la racionalidad a la hora de comprar es patrimonio casi exclusivo de los hombres. Asímismo, López y Schneider (2007) en un estudio realizado en la Universidad de La Frontera, señalan que las mujeres poseen un endeudamiento levemente mayor por sobre el de los hombres.

Otra variable a considerar en el estudio de actitudes hacia el ahorro, compra y endeudamiento en jóvenes estudiantes y titu- 
lados universitarios es el nivel socioeconómico. Diversos autores plantean la existencia de prácticas transversales como el consumo reflexivo y el uso de tarjeta de crédito como método principal de endeudamiento (Ariztía, Melero \& Montero, 2010; Denegri, Cabezas, del Valle, González \& Sepúlveda, 2012); sin embargo, se ha descrito que en los niveles socioeconómicos altos existen mayores índices de ahorro, asociados justamente al ingreso real per cápita (Loayza, Schmidt-Hebbel \& Servén, 2001), lo que apoyaría lo reportado por Denegri et al. (2012), quienes indican que el tipo de deuda y el nivel de endeudamiento en estudiantes universitarios difieren significativamente según el nivel socioeconómico.

En cuanto a la formación académica asociada al comportamiento económico, es importante señalar que algunas carreras ligadas a los ámbitos de la economía, negocios o administración pueden entregar ciertas herramientas para desenvolverse con ventaja en estos ámbitos como lo demuestran estudios en Israel y Uruguay (Caffera, Zipitría \& Arboleya, 2011); sin embargo, en un estudio realizado con jóvenes universitarios chilenos (Medina et al., 1999) señala que no habría diferencias significativas en las actitudes hacia el endeudamiento ni en función de la facultad a la que pertenecían ni del género de los participantes. De manera similar, un estudio con universitarios colombianos (Amar, Abello, Denegri, \& Llanos, 2007) no se encontraron diferencias significativas entre variables como valor y circulación del dinero, emisión y acuñación monetaria respecto al programa de formación académica de distintos estudiantes de carreras de Derecho, Psicología e Ingeniería.

En consideración de lo expuesto, resulta relevante explorar las actitudes que personas inmersas en un modelo económico neoliberal sostienen en cuanto a ahorro, compra, consumo y endeudamiento, especial mente en el grupo de titulados de educación superior.

\section{Pregunta, objetivos de investigación e hipótesis}

Considerando los antecedentes empíricos expuestos, el objetivo de la presente investigación fue comparar las actitudes hacia el ahorro, el consumo y el endeudamiento en titulados de una universidad pública del sur de Chile, a partir de su género, nivel socioeconómico y facultad de procedencia. Para ello, se buscó responder la siguiente pregunta de investigación: ¿Existen diferencias en cuanto a las actitudes hacia el ahorro, consumo y endeudamiento en titulados de una universidad pública del sur de Chile, a partir de su género, nivel socioeconómico y facultad de procedencia? Para responder a la pregunta de investigación se plantea como objetivos específicos: (1) caracterizar las actitudes hacia el ahorro, consumo y endeudamiento de los participantes; (2) identificar si existen diferencias significativas entre las actitudes hacia el ahorro, consumo y endeudamiento a partir de su género, nivel socioeconómico y facultad de procedencia; (3) caracterizar las relaciones entre actitudes hacia el consumo, ahorro y endeudamiento de los participantes.

\section{Método}

\section{Diseño y procedimiento}

El presente estudio contó con un diseño cuantitativo, no experimental, ex post facto, de alcance descriptivo-correlacional y de tipo transversal (Hernández, Fernández \& Baptista, 2010). Los participantes fueron contactados mediante el método bola de nieve, invitándolos a responder una serie de instrumentos online, y en los casos en que los participantes lo solicitaron, se les hizo llegar una versión impresa. En ambos casos, se les enviaba el formulario de consentimiento informado donde se señalaba la voluntariedad de su participación y el anonimato y confidencialidad de la información provista. El estudio no contempló gratificación material o econó- 
mica. La recogida de datos se llevó a cabo en un período de 6 meses.

\section{Participantes}

En el presente estudio se utilizó un muestreo no probabilístico mediante el método bola de nieve ${ }^{1}$, alcanzando un total de 120 participantes (hombres: 46.7\%; mujeres: $53.3 \%$ ) titulados de una universidad pública del sur de Chile en los últimos 7 años. En razón de la facultad de la que egresaron, se distribuyeron de la siguiente forma: 72 de la Facultad de Educación, Ciencias Sociales y Humanidades (60\%); 13 de la Facultad de Ciencias Agropecuarias y Forestales (10.8\%); 22 de la Facultad de Ingeniería, Ciencias y Administración (18.3\%) y 13 de la Facultad de Medicina (10.8\%), siendo las últimas tres reagrupadas en una sola categoría con el fin de crear dos grupos de comparación más o menos equivalentes. En cuanto a nivel socioeconómico, 57 participantes pertenecían al nivel socioeconómico alto $(47.5 \%)$ y 63 al nivel socioeconómico medio $(52.5 \%)$. La edad promedio de los participantes era 27.1 años $(D E=3.4)$.

\section{Instrumentos}

Los instrumentos administrados fueron la Escala de Actitudes hacia el Ahorro, la Escala de Actitudes hacia el Endeudamiento y la Escala de Actitudes hacia la Compra. Se incluyeron además preguntas de clasificación sociodemográfica y la Escala de Evaluación del Nivel Socioeconómico ESOMAR (ADIMARK, 2000). Estos instrumentos fueron seleccionados por su amplio uso en investigaciones previas en población chilena, arrojando en distintas oportu-

\footnotetext{
${ }^{1}$ Originalmente, el estudio contemplaba muestreo por autoselección (voluntarios) con establecimiento de cuotas según género, nivel socioeconómico y facultad de los titulados, con el objetivo de obtener una muestra representativa. Sin embargo, debido a complicaciones con el acceso a la muestra, se optó por un muestreo mediante bola de nieve.
}

nidades niveles aceptables de confiabilidad y validez (e.g. Denegri et al., 1999; Otto \& Webley, 2009; Luna-Arocas \& Fierres, 1998).

La Escala de Actitudes hacia el Ahorro (Otto \& Webley, 2009) permite evaluar las actitudes hacia el ahorro a partir de 30 ítems, midiendo las tendencias actitudinales hacia el ahorro y tres aspectos del ahorro: el ahorro como algo necesario, el ahorro como algo positivo y el ahorro como algo difícil de conseguir. La presente investigación utilizó una versión abreviada de 15 ítems en formato Likert que va desde 1 (completamente en desacuerdo) hasta 6 (completamente de acuerdo). Los índices de confiabilidad $\alpha$ para esta escala en estudios previos fueron entre .66 y .78, según lo reportado por Otto y Webley (2009), mientras que en el estudio que se informa, el índice de confiabilidad $\alpha$ fue .75 .

La Escala de Actitudes hacia el Endeudamiento (Denegri et al., 1999) permite evaluar la actitud de las personas frente a las deudas que posee como individuo. Contiene dos subescalas: una mide estilo actitudinal austero (propensión hacia el uso cauto del dinero, orientación hacia el ahorro y evitación del crédito) y otra mide estilo actitudinal hedonista (tendencia hacia uso del dinero como medio para el logro de satisfacciones, orientación hacia el uso de crédito $\mathrm{y}$ al endeudamiento). La escala total consta de 11 ítems, 7 corresponden a la subescala de austeridad y 4 a la de hedonismo, en formato de respuesta tipo Likert que va desde 1 (completamente en desacuerdo) hasta 6 (completamente de acuerdo). Estudios previos (CEPEC \& CDS, 2012) reportan índices aceptables de confiabilidad para las subescalas de austeridad y hedonismo $(\alpha=.89$ y $\alpha=.57$, respectivamente). En la presente investigación, los índices resultaron levemente más bajos, aunque aún dentro del rango aceptable $(\alpha=.75$ para austeridad y $\alpha=.65$ para hedonismo).

La Escala de Actitudes hacia la Compra (Luna-Arocas \& Fierres, 1998) está compuesta por tres subescalas que evalúan creencias y estilos de consumo y actitud hacia 
la compra: racionalidad, compulsividad e impulsividad en la compra. La escala consta de 18 ítems de los cuales 4 corresponden a la subescala de racionalidad, 7 a la de compulsividad y 7 a la de impulsividad en la compra. Los ítems siguen un formato de respuesta tipo Likert que va desde 1 (completamente en desacuerdo) hasta 6 (completamente de acuerdo). Estudios previos reportan coeficientes de confiabilidad adecuados: .83 para racionalidad, .91 para compulsividad y .79 para impulsividad en la compra (CEPEC \& CDS, 2012). En la presente investigación se obtuvieron coeficientes similares: .83 para racionalidad, .92 para compulsividad y .82 para impulsividad en la compra.

\section{Plan de análisis}

En primera instancia se realizaron análisis descriptivos con el fin de caracterizar la muestra en cuanto a variables sociodemográficas y a sus respuestas en las escalas de actitudes hacia el endeudamiento, el ahorro y el consumo. Se calcularon los índices de confiabilidad para cada una de las escalas y subescalas de actitudes mediante Alpha de Cronbach, se evaluó la distribución normal de las variables para cada subgrupo mediante la prueba Kolmogorov-Smirnov y se realizaron pruebas de comparación de medias y medianas para dos muestras independientes según correspondiera para las comparaciones según género, nivel socioeconómico y facultad de procedencia (pruebas $t$ para muestras independientes y pruebas $U$ de Mann-Whitney). Para identificar posibles asociaciones entre las distintas escalas y subescalas, se realizaron pruebas de correlación $r$ de Pearson.

\section{Resultados}

Para responder al objetivo específico que busca caracterizar las actitudes hacia el ahorro, el consumo y el endeudamiento de los participantes, se calculó la media de las puntuaciones obtenidas por los participan- tes en las diferentes escalas y subescalas. Puntuaciones sobre el punto medio de la escala (3.50) fueron consideradas como actitudes favorables, mientras que debajo de ese punto fueron consideradas desfavorables.

En cuanto al ahorro, la muestra presenta una actitud favorable $(M=4.45 ; D E=.60)$, indicando una tendencia a evaluar positivamente la acumulación de recursos propios en beneficio de un uso futuro a mediano o largo plazo.

Con respecto al endeudamiento, los participantes presentan una actitud desfavorable hacia el endeudamiento hedónico $(M=$ $3.03 ; D E=1.00$ ), indicando una tendencia a evaluar negativamente el endeudamiento con fines de satisfacer necesidades momentáneas. En referencia a la subescala de austeridad, la muestra presenta una actitud favorable hacia el endeudamiento responsable, concienzudo y sistematizado $(M=$ $5.23 ; D E=.73$ ), lo que a la larga favorecería la situación financiera de quien planifica sus compras.

Los participantes demostraron una actitud favorable hacia la compra racional $(M=$ $4.07 ; D E=1.18$ ), indicando una tendencia a evaluar positivamente la planificación y seguridad al consumir. Asimismo, demostraron una actitud desfavorable hacia la compra impulsiva $(M=3.28 ; D E=.99)$, indicando una evaluación negativa hacia la compra mediada por el impulso y sin planificación, y hacia la compra compulsiva $(M=1.88$; $D E=1.02$ ), evidenciando una actitud desfavorable hacia compras que no se asocian a una necesidad real, pero que demandan una excesiva preocupación por comprarlas por parte de las personas. Para un resumen de las puntuaciones medias en las diferentes subescalas, referirse a la Figura 1.

Para responder al segundo objetivo específico que busca identificar si existen diferencias significativas entre las actitudes hacia el ahorro, el consumo y el endeudamiento a partir del género, nivel socioeconómico y facultad de procedencia de los participantes, se realizaron comparaciones de medias para datos paramétricos y no pa- 
ramétricos, con pruebas $t$ para muestras independientes y $U$ de Mann-Whitney, respectivamente. Como paso previo, se evaluó la distribución normal de dichas variables a través de la prueba Kolmogorov-Smirnov ( $p \leq$ $.05)$.

En cuanto a las comparaciones según nivel socioeconómico, es preciso mencionar que la escala utilizada para medirlo utiliza para su cálculo el nivel educacional y el tipo de trabajo de quien aporta el principal ingreso económico en el hogar. Considerando que esta investigación consideró solo titulados universitarios, el nivel socioeconómico tendió a los grupos alto, medio-alto y medio, los que debieron ser recodificados en dos grupos (alto y medio) para poder hacer estas comparaciones con grupos equivalentes en tamaño, pues no se contaban con suficientes sujetos en todos los subgrupos para hacer los análisis. De manera similar, para facilitar las comparaciones según la facultad de origen de los participantes, se recodificó en dos grupos: titulados en la Facultad de Educación, Ciencias Sociales y Humanidades y titulados en otras facultades (Ingeniería, Ciencias y Administración, Medicina y Ciencias Agropecuarias y Forestales). Esta recodificación se realizó a fin de contar con grupos de comparación más equilibrados en cuanto a su tamaño.

La escala de actitudes hacia el ahorro se ajusta a una distribución normal $(D(120)=$ $.073 ; p=$ n.s.), sin encontrarse diferencias estadísticamente significativas según género $\left(t_{(118)}=-.61 ; p=\right.$ n.s. $)$, ni según nivel socioeconómico $\left(t_{(118)}=-.72 ; p=\right.$ n.s. $)$, ni según facultad de procedencia $\left(t_{(119)}=-.52\right.$, $p=$ n.s.).

La escala de actitudes hacia el endeudamiento, subescala de hedonismo, no se ajusta a una distribución normal $(D(120)=$ $.082 ; p<.05)$ sin encontrarse diferencias estadísticamente significativas según género $(U=1690 ; p=$ n.s. $)$, nivel socioeconómico ( $U=16190,5 ; p=$ n.s. $)$, ni facultad de procedencia $(U=1561 ; p=$ n.s.). En forma similar, la subescala de austeridad tampoco se ajusta a una distribución normal $(D(120)=$
$.183 ; p<.001)$. Al comparar por nivel socioeconómico, se encontraron diferencias significativas $(U=1353,5 ; p<.01)$, donde los participantes de nivel socioeconómico alto obtuvieron puntuaciones más altas $(M e=5.57 ; D E=.58)$ que los participantes de nivel socioeconómico medio $(M e=5.29$; $D E=.84$ ), aunque el tamaño del efecto para dicha diferencia fue pequeño $\left(\eta^{2}=.01\right)$. No se encontraron diferencias estadísticamente significativas según género $(U=1718 ; p=$ n.s.) ni facultad de procedencia $(U=1622$; $p=$ n.s.).

Respecto a la escala de actitudes hacia la compra, la subescala de racionalidad en la compra no se ajusta a una distribución normal $(D(120)=.082 ; p<.05)$. No se encontraron diferencias estadísticamente significativas según género ( $U=1502 ; p=$ n.s. $)$, nivel socioeconómico $(U=1776 ; p=$ n.s. $)$, ni facultad de procedencia $(U=1585.5 ; p=$ n.s.). En cuanto a la subescala de impulsividad en la compra, las puntuaciones se ajustan a una distribución normal $(D(120)=$ $.072 ; p=$ n.s. $)$, sin encontrarse diferencias significativas por género $\left(t_{(118)}=-1.07 ; p=\right.$ n.s.), nivel socioeconómico $\left(t_{(118)}=.58 ; p=\right.$ n.s. $)$, ni facultad de procedencia $\left(t_{(119)}=-\right.$ $1.183 ; p=$ n.s.). Finalmente, en la escala de compulsividad en la compra, las puntuaciones no se ajustan a una distribución normal $(D(120)=.194 ; p<.001)$, encontrando diferencias estadísticamente significativas al comparar por género de los participantes $(U=1380 ; p<.05)$, donde las mujeres obtuvieron puntuaciones significativamente más altas que los hombres $(M e=1.79, D E=$ $1.01 ; M e=1.29, D E=1.04$, respectivamente), aunque el tamaño del efecto para dicha diferencia fue pequeño $\left(\eta^{2}=.04\right)$. No se hallaron diferencias estadísticamente significativas según nivel socioeconómico $(U=$ $1537 ; p=$ n.s.) ni facultad de procedencia $(U=1605 ; p=$ n.s. $)$.

Para responder al objetivo específico que busca caracterizar las relaciones entre actitudes hacia el consumo, ahorro y endeudamiento de los participantes, se realizó la prueba $r$ de Pearson (ver Tabla 1), que resultó significativa en las siguientes correla- 
ciones: actitudes hacia el ahorro y austeridad en la compra $(r=.19 ; p<.05)$, y actitudes hacia el ahorro y racionalidad en la compra $(r=.21 ; p<.05)$-en ambos casos con correlaciones débiles-, entre compulsividad en la compra y austeridad con una correlación moderada $(r=-.32 ; p<.001)$, y entre compulsividad en la compra e impulsividad en la compra con una correlación fuerte $(r=.66 ; p<.001)$.

\section{Discusión}

El objetivo general del presente estudio fue comparar las actitudes hacia el ahorro, consumo y endeudamiento en titulados de una universidad pública del sur de Chile, a partir de su género, nivel socioeconómico y facultad de procedencia. Para la consecución de dicho objetivo general se plantearon tres objetivos específicos cuyos resultados se discutirán a continuación.

Respondiendo al primer objetivo específico, es posible destacar los niveles favorables de actitudes hacia el ahorro, hacia la subescala de austeridad (actitudes hacia el endeudamiento) y hacia la racionalidad en la compra (actitudes hacia el consumo), y desfavorables hacia el hedonismo (actitudes hacia el endeudamiento), compulsividad e impulsividad en la compra (actitudes hacia la compra). Estos resultados permiten caracterizar a esta muestra como individuos que, en general, tienden a ahorrar su dinero, invertirlo racionalmente en bienes o servicios y endeudarse solo en caso que resulte necesario.

Los resultados para este primer objetivo específico se contraponen con los indicadores que entrega el INJUV (2003, 2007, 2009), que señalan que los jóvenes son uno de los sectores etarios más endeudados del país. Esta diferencia podría apuntar a una diferencia entre las actitudes reportadas hacia el ahorro, compra y endeudamiento (como fueron evaluadas en el presente estudio), versus lo reportado por los sujetos en medidas más directas y/u objetivas (como fue el caso de los estudios del INJUV). Esta discrepancia puede deberse a una disonan- cia actitudinal/conductual y/o al efecto que la deseabilidad social pudiera haber tenido en las respuestas de los sujetos en la presente investigación, cuya no consideración constituye una de sus limitaciones.

Respondiendo al segundo objetivo específico, es posible concluir que no existen diferencias de género en las actitudes hacia el ahorro, actitudes hacia el endeudamiento (austeridad y hedonismo) y la compra (racionalidad e impulsividad en la compra). Sí se encontraron diferencias significativas en cuanto al género para la subescala de compulsividad en la compra, similar a lo reportado por López y Schneider (2007). Este resultado apunta a que las mujeres tendrían actitudes más positivas hacia el consumo compulsivo, aunque, considerando el tamaño del efecto, dicha diferencia es leve.

Con respecto a las comparaciones según nivel socioeconómico, se encontraron diferencias en la subescala de austeridad, lo que indicaría que el nivel socioeconómico alto presenta una mayor tendencia al uso cauto del dinero, orientado hacia el ahorro y evitación del crédito, versus el nivel socioeconómico medio. Estos resultados corroboran lo reportado por la 6ta Encuesta Nacional de la Juventud (INJUV, 2009) donde se reportan diferencias en el endeudamiento entre quienes poseen ingresos propios en el nivel socioeconómico medio y alto, en la misma dirección que la presente investigación. Una posible explicación para estos resultados se refiere a la capacidad de los niveles socioeconómicos altos para pagar sin necesidad de endeudarse, lo que terminaría internalizándose como una actitud más favorable hacia la austeridad. En contraste, el nivel socioeconómico medio, que también aspira a similares objetos y beneficios que el nivel alto, se vería en la necesidad de gastar más allá de sus recursos monetarios (Denegri et al., 2012), lo que generaría la internalización de actitudes menos positivas hacia la austeridad como justificación a su propio comportamiento.

Por otro lado, no hubo diferencias estadísticamente significativas entre las escalas de actitudes respecto de la facultad de egreso de los titulados. Esto coincide con lo reportado por Medina et al. (1999), quienes 
reportan no haber encontrado diferencias significativas entre estudiantes universitarios de diferentes carreras y facultades en cuanto a sus actitudes hacia el endeudamiento y sus subescalas. Esto permitiría concluir que la formación que reciben los jóvenes universitarios no afectaría sus actitudes hacia el ahorro, consumo y endeudamiento, más allá de los conocimientos teóricos que pudieran recibir. Estos resultados coinciden con los de Amar et al. (2007), quienes no encontraron diferencias significativas entre variables como valor y circulación del dinero, emisión y acuñación monetaria respecto al programa de formación académica de distintos estudiantes de carreras de Derecho, Psicología e Ingeniería, y también por lo reportado por Sepúlveda et al. (2017), que informan que la educación formal tendría un impacto más bien limitado en cuanto a alfabetización económica, lo que indicaría la necesidad de fortalecer la malla curricular de carreras universitarias para propender a la entrega de contenidos teóricos y al desarrollo de comportamientos económicos responsables, racionales, que los preparen para la inserción al mundo laboral (Denegri, Martínez \& Etchebarne, 2007) y su participación activa como ciudadanos (Amar et al., 2007).

Respondiendo al tercer objetivo específico, es posible concluir que la actitud hacia el ahorro está asociada con la austeridad (subescala de actitudes hacia el endeudamiento) y con la racionalidad en la compra (subescala de actitudes hacia la compra). De manera similar, la compulsividad en la compra (subescala de actitudes hacia la compra) está inversamente asociada con la austeridad (subescala de actitudes hacia el endeudamiento) y directamente asociada con la impulsividad en la compra (subescala de actitudes hacia la compra). Estos resultados coinciden con el sustento teórico de las escalas, pues es de esperar que una actitud positiva hacia el ahorro esté asociada con un comportamiento tendiente al uso cauto y racional del dinero, orientado hacia el ahorro y evitación del crédito, mientras que un consumo compulsivo estaría asociado a un consumo altamente dependiente de las claves contextuales, variables situa- cionales y un uso poco planificado de herramientas de endeudamiento.

En resumen, estos resultados permiten concluir un perfil esperable en cuanto a actitudes hacia el ahorro, endeudamiento y la compra, en profesionales universitarios, aunque no queda claro hasta qué punto dichos resultados se pudieran deber a la influencia de la deseabilidad social en las respuestas de los participantes. Si bien se encontraron algunas diferencias significativas en cuanto a género y nivel socioeconómico de los participantes, estas diferencias son más bien bajas, lo que puede deberse a la homogeneidad de la muestra en cuanto a nivel socioeconómico y experiencias de vida asociadas a la formación universitaria y vida profesional. Finalmente, se hallaron asociaciones entre actitudes hacia el ahorro con austeridad y con racionalidad en la compra. También se encontraron asociaciones entre compulsividad en la compra con austeridad (inversamente) y con impulsividad en la compra, lo que apunta a una coherencia teórica de los constructos y sus dimensiones.

Estos hallazgos sugieren la necesidad de fortalecer la formación actitudinal de los estudiantes universitarios y futuros profesionales en cuanto a alfabetización económica. Esta implica la adquisición de conductas económicas racionales y prepara a los estudiantes universitarios para la inserción en el mundo laboral (Denegri et al., 2007) y para participar activamente como ciudadano (Amar et al., 2007). Resulta crucial que los jóvenes universitarios reciban esta formación considerando que, tras su titulación y muchas veces la obtención de su primer empleo, se hacen cargo de su propia solvencia económica sin supervisión formal de los padres. En este contexto, actitudes negativas hacia el ahorro, la austeridad y el consumo racional podrían implicar consecuencias sociales y legales asociadas al endeudamiento y consumo que pueden arrastrar por años.

Una primera sugerencia para futuras investigaciones es incorporar mediciones externas respecto al comportamiento de los sujetos en cuanto a ahorro, endeudamiento y compra. Al incorporar estas mediciones 
sería posible comparar el aspecto actitudinal con el comportamental, pudiendo establecer de esta forma en qué medida las actitudes son un buen predictor del comportamiento de los sujetos en el medio real. De manera similar, se sugiere incorporar una medida de deseabilidad social que permita esclarecer en qué medida las respuestas de los sujetos están siendo influenciadas por la deseabilidad social.

Una de las limitaciones del estudio es la relativa homogeneidad de la muestra, que se ve reflejada en la distribución por nivel socioeconómico tendiente a niveles altos y que se explicaría al menos en parte por el método de muestreo utilizado. Otra limitación proviene del uso de medidas indirectas (eg. escalas actitudinales) susceptibles a deseabilidad social de los participantes en vez de medidas objetivas/directas. Por otra parte, al utilizar un muestreo no-probabilístico por bola de nieve, se estaría violando el supuesto de aleatoriedad necesario para la utilización de pruebas $t$, lo que afectaría la validez de los resultados de estos análisis. Finalmente, la limitación correspondiente al uso de un diseño correlacional, junto a las limitaciones ya mencionadas, dificultaría la generalización de los resultados y su comparación con otras muestras.

Con estas limitaciones en consideración, se sugiere que futuros estudios amplíen el espectro de la muestra mediante la utilización de un método de muestreo aleatorio, aumentando el tamaño de la muestra y estableciendo cuotas equivalentes en tamaño por carreras y/o facultades. Esta sugerencia busca enriquecer la comprensión de las actitudes económicas en titulados universitarios, más allá de la experiencia de un grupo delimitado de participantes, como pudiera ser el caso de la presente investigación. Para la implementación de estas sugerencias es necesario contar con el apoyo institucional para contactar a los titulados a través de vías formales de comunicación, a la vez que se diversifica el grupo de posibles participantes. Por otra parte, el establecimiento de cuotas equivalentes por carrera y/o facultad permitiría la comparación de dichos grupos sin la necesidad de reagruparlos en nuevas categorías y la consecuente pérdida de información que conlleva dicha recodificación.

Tabla 1

\section{Confiabilidad y correlaciones}

\begin{tabular}{|c|c|c|c|c|c|c|}
\hline & 1 & 2 & 3 & 4 & 5 & 6 \\
\hline 1- Actitud hacia el ahorro & - & -.15 & $.19^{*}$ & $.21^{*}$ & -.07 & -.12 \\
\hline $\begin{array}{l}\text { 2- Actitud hacia el endeudamiento } \\
\text { Subescala: Hedonismo }\end{array}$ & & - & .04 & .02 & .08 & -.03 \\
\hline $\begin{array}{l}\text { 3- Actitud hacia el endeudamiento } \\
\text { Subescala: Austeridad }\end{array}$ & & & - & .08 & -.08 & $-.32^{* * * *}$ \\
\hline $\begin{array}{l}\text { 4- Actitud hacia el consumo } \\
\text { Subescala: Racionalidad }\end{array}$ & & & & - & .02 & -.04 \\
\hline $\begin{array}{l}\text { 5- Actitud hacia el consumo } \\
\text { Subescala: Impulsividad }\end{array}$ & & & & & - & $.66^{* * * *}$ \\
\hline \multirow[t]{2}{*}{$\begin{array}{l}\text { 6- Actitud hacia el consumo } \\
\text { Subescala: Compulsividad }\end{array}$} & & & & & & - \\
\hline & .75 & .65 & .75 & .83 & .82 & .92 \\
\hline
\end{tabular}

* Correlación significativa a nivel .05.

*** Correlación significativa a nivel .001. 


\section{Referencias bibliográficas}

ADIMARK. (2000). El nivel socio económico ESOMAR. Manual de aplicación. Santiago, Chile: GfK Adimark.

Amar, J., Abello, R., Denegri, M. \& Llanos, M. (2007). Pensamiento económico en jóvenes universitarios. Revista Latinoamericana de Psicología, 39(2), 363-373.

Ariztía, T., Melero, J. \& Montero, J. (2010). Un nuevo consumidor chileno: de los derechos a las responsabilidades. Santiago, Chile: Fundación Ciudadano Responsable.

Armanet, P. (2005) Formación universitaria para el siglo XXI. Expansiva, Serie En Foco, 44, 1-10. Recuperado el 17 de marzo de 2016 de http://www.expansiva. cl/publicaciones/en_foco/detalle.tpl?iddocumento $=09052005141513$

Ayllón, M., Vallejos, P. \& Yáñez, V. (1999). Hábitos y conducta de consumo de los estudiantes de la Universidad de La Frontera. (Tesis de pregrado no publicada). Universidad de La Frontera, Temuco, Chile.

Baeza, J., Durán, R. \& Vargas, C. (1999). Psicogénesis de conceptos económicos, según nivel de formación y género, en estudiantes de cuarto y quinto año de la Universidad de La Frontera. (Tesis de pregrado no publicada). Universidad de La Frontera, Temuco, Chile.

Borrás, V. (2007). Las desigualdades en el consumo a través del Género. Revista Española de Sociología, 8, 139-156.

Caffera, M., Zipitría, L. \& Arboleya, L. (2011). ¿Estudiar economía te hace más egoísta? Revista de Ciencias empresariales y economía, 24, 29-35.

Carrillo, J. (2010). El neoliberalismo en Chile: entre la legalidad y la legitimidad. Entrevista a Tomás Moulián. Perfiles Latinoamericanos, 18(35), 145-155.

Catalán, O. (2010) Juventud y consumo: Bases analíticas para una problematización.
Última Década, 32, 137-158. http://dx. doi. org/10.4067/S0718-22362010000100008

CEPEC \& CDS. (2012). Perfiles de los consumidores del gran Temuco, informe ejecutivo. Proyecto fondo concursable SERNAC, para asociaciones de consumidores. Temuco, Chile: Universidad de La Frontera.

Denegri, M. (1998). La construcción de nociones económicas en la infancia y adolescencia. En J. Ferro, \& J. Amar (Eds.), Desarrollo humano: Perspectiva. Barranquilla. Colombia: Siglo XXI, Ediciones UNINORTE.

Denegri, M. \& Martínez, G. (2004). ¿Ciudadanos o consumidores? Aportes constructivistas a la educación para el consumo. PAIDEIA, Revista de Educación, 37, 101116.

Denegri, M., Cabezas, D., del Valle, C., González, Y. \& Sepúlveda, J. (2012). Escala de Actitudes hacia el Endeudamiento: validez factorial y perfiles actitudinales en estudiantes universitarios chilenos. Universitas Psychologica, 11(2), 497-509.

Denegri, M., Gempp, R., Del Valle, C., Etchebarne, S. \& González, Y. (2006). El aporte de la psicología educacional a las propuestas de educación económica: los temas claves. Revista de Psicología de la Universidad de Chile, 15(2), 69-84. http:// dx.doi.org/10.5354/0719-0581.2006.18398

Denegri, M., Iturra, R., Palavecinos, M. \& Ripoll, M. (1999). Consumir para vivir y no vivir para consumir. Temuco: Ediciones Universidad de La Frontera.

Denegri, M., Martínez, G. \& Etchebarne, S. (2007). La comprensión del funcionamiento bancario en adolescentes chilenos: Un estudio de Psicología Económica. Interdisciplinaria, 24(2), 137-159.

Duarte, C. (2009) Sobre los que no son, aunque sean. Éxito como Exclusión de Jóvenes Empobrecidos en Contextos Capitalistas. Última Década, 30, 11-39. http://dx.doi. org/10.4067/S0718-22362009000100002 
Espinoza, O. \& González, L. (2010). Movilidad Social en Chile: El caso del gran Santiago urbano. Artículos de revistas CIE, 1, 2-26.

Hernández, R., Fernández, C. \& Baptista, P. (2010). Metodología de la investigación. México D.F.: McGraw Hill.

Instituto Nacional de la Juventud. (2003). $4^{a}$ Encuesta Nacional de Juventud. Recuperado el 17 de marzo de 2016 de http://www. injuv.gob.cl/portal/categoria/publicaciones/encuestas-de-juventud/

Instituto Nacional de la Juventud. (2007). $5^{a}$ Encuesta Nacional de Juventud. Recuperado el 17 de marzo de 2016 de http://www.injuv. gob.cl/portal/categoria/publicaciones/encuestas-de-juventud/

Instituto Nacional de la Juventud. (2009). $\sigma^{a}$ Encuesta Nacional de Juventud. Recuperado el 17 de marzo de 2016 de http://www.injuv. gob.cl/portal/categoria/publicaciones/encuestas-de-juventud/

Lea, S., Webley, P. \& Levine, R. (1993). The economic psychology of consumer debt. Journal of Economic Psychology, 14, 85119. https://doi.org/10.1016/0167-4870(93) 90041-I

Lipovetsky, G. (2002). La Era del Vacio. Barcelona, España: Ediciones Anagrama.

Loayza, N., Schmidt-Hebbel, K. \& Servén, L. (2001). Una revisión del comportamiento y de los determinantes del ahorro en el mundo. Banco Central de Chile, Documentos de trabajo, $\mathrm{N}^{\circ} 95$.

López, L. \& Schneider, I. (2007). Los nuevos instrumentos crediticios para jóvenes en Chile: tarjetas de crédito de casas comerciales e instituciones bancarias, un análisis del nivel de endeudamiento de los alumnos de la Universidad de la Frontera. (Tesis de pregrado no publicada). Universidad de la Frontera, Temuco, Chile.

Luna-Arocas, R. (1998). Dinero, trabajo y consumo. Valencia, España: Promolibro.

Luna-Arocas, R. \& Fierres, R. (1998). La incidencia de la compra por impulso en la ciu- dad de Valencia. Investigación y Marketing, 60, 36-42.

McElprang, C., Haskell C. \& Jenkins, J. (2009). Financial attitudes and spending habits of university freshmen. Journal of Economics and Economic Education, 10(1), 3-20.

Medina, M., Méndez, G., Pérez, C. \& Quezada, J. (1999). Evaluación de conductas y actitudes hacia el endeudamiento en estudiantes de la Universidad de La Frontera. (Tesis de pregrado no publicada). Universidad de La Frontera, Temuco, Chile.

Meneses, F., Rolando, R., Valenzuela, M. \& Vega, M.A. (2010) Ingreso a la Educación Superior: la experiencia de la cohorte de egreso 2005. Santiago, Chile: Sistema Nacional de Información de la Educación Superior (SIES) y División de Educación Superior del Ministerio de Educación (MINEDUC) de Chile.

Modigliani, F. (1966). The life cycle of saving, the demand for wealth and the supply of capital. Social Research, 33(2), 323-381.

Modigliani, F. (1986). Life cycle, individual thrift, and the wealth of nations. American Economic Review, 76(3), 297-313.

Ortiz, C.H. (2002). Economic structure, international income differentials and long-run growth. Revista de Análisis Económico, 17(1), 45-70.

Otto, A. \& Webley, P. (2009). ;Hey pequeño consumidor! Pronosticadores psicológicos del ahorro adolescente. Streatham, England: University of Exeter.

PNUD/INJUV. (2003). Transformaciones culturales e identidad juvenil en Chile. Santiago, Chile: PNUD/INJUV.

Sepúlveda, J., Denegri, M., Orellana, L., Criado., N., Mendoza, J., Salazar, P. \& Yung, G. (2017). Características Emprendedoras Personales y Alfabetización Económica: Una Comparación en Estudiantes Universitarios del Sur de Chile. Interdisciplinaria, 34(1), 107-124. https://doi.org/10. 16888/interd.2017.34.1.7 
Tironi, E. (1999). La irrupción de las masas y el malestar de las elites: Chile en el cambio de siglo. Santiago, Chile: Grijalbo.

Toro, M. (2014, Mayo 12). Créditos bancarios para costear educación superior crecen en $19 \%$ al año, superando alza de préstamos hipotecarios y de consumo. El Mercurio, p. 6.
Vidal, D. (1997) La compra de símbolos sociales frente al consumo de objetos. Revista de Sociología, 51, 35-57. http://dx.doi.org/10. 5565/rev/papers. 1856

Recibido: 9 de marzo de 2017 Aceptado: 3 de septiembre de 2018 
